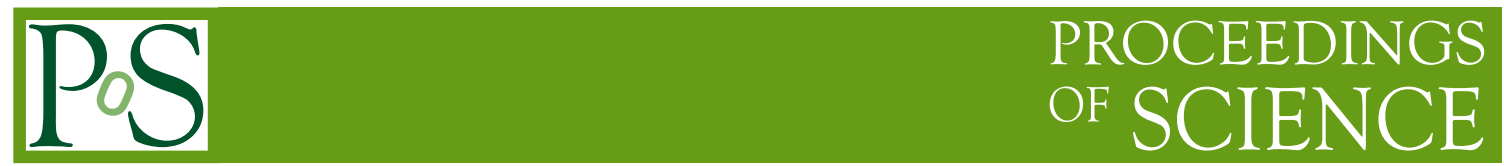

\title{
Results from $\mathrm{LHCb}$ in charmless (quasi)-two body $B$ meson decays
}

\author{
Sebastiana Giani* ${ }^{*}$ \\ Ecole polytechnique fédérale de Lausanne (EPFL) \\ E-mail: sebastiana.giani@epfl.ch
}

Charmless $b$-hadron decays are a good probe for testing the Standard Model. Moreover, as they can proceed through loop diagrams, they are also sensitive to physics beyond the Standard Model. We report recent results from LHCb on charmless $b$-hadron two-body and quasi-two-body decays.

9th International Workshop on the CKM Unitarity Triangle

28 November - 3 December 2016

Tata Institute for Fundamental Research (TIFR), Mumbai, India

* Speaker.

${ }^{\dagger}$ on behalf of the LHCb Collaboration 


\section{Introduction}

Charmless hadronic $B$ decays are important for testing the Standard Model (SM) and studying $\mathrm{CP}$ violation. In addition, precision measurements of these decays are useful to search for physics beyond the Standard Model (BSM). Indeed, for charmless $B$ decays the amplitude of the first order tree-level diagrams depends on the $V_{u b}$ CKM element. This element being very small, loop diagrams can become competitive. Hence BSM physics could enter in the loop diagrams and give a sizable contribution to several observables [1]. The branching fractions for charmless modes are of the order of $10^{-6}-10^{-5}$, which is small with respect to the Cabbibo-favoured $b \rightarrow c$ transition. However this order of branching fraction is still large enough to be studied at LHCb, although this strongly depends on the final state and on the reconstruction efficiency. Indeed the LHCb geometry is optimised for the detection of hadrons containing $b$ quarks. At high-energy $p p$ collisions, the $b \bar{b}$ quark pairs are predominantly produced in the same forward or backward cone. The large $b \bar{b}$ production cross section, $75.3 \pm 5.4 \pm 13.0 \mu \mathrm{b}$ at $7 \mathrm{TeV}$ [2], allows the collection of large statistics samples of these decays. The excellent performance of the LHCb detector in the tracking, trigger and particle identification allows an efficient reduction of the abundant background present in the hadron collider environment. In these proceedings, we report three recent measurements from the LHCb experiment, all based on the full set of data of $3 \mathrm{fb}^{-1}$ collected at centre-of-mass energies of 7 and $8 \mathrm{TeV}$ in 2011 and 2012, respectively.

\section{2. $B^{0} \rightarrow K^{+} K^{-}$and $B_{s}^{0} \rightarrow \pi^{+} \pi^{-}$}

The $B^{0} \rightarrow K^{+} K^{-}$decay is the rarest mode in the family $B \rightarrow h h$ (with $h=\pi, K$ ) and the rarest fully hadronic $B$-meson decay ever observed [3]. In the SM, this rare mode, together with $B_{s}^{0} \rightarrow \pi^{+} \pi^{-}$, proceeds predominantly through weak annihilation transitions. While the $B_{s}^{0} \rightarrow \pi^{+} \pi^{-}$ has already been observed by LHCb [4], the $B^{0} \rightarrow K^{+} K^{-}$was never observed so far. The selected candidates must satisfy hardware and software trigger requirements. The former implies having large transverse energy clusters in the hadronic calorimeter, and the latter a well reconstructed and displaced secondary vertex (SV) and at least one charged track with a large impact parameter (IP) with respect to any of the reconstructed $p p$ interaction points. The trigger efficiency is significantly improved thanks to an exclusive selection algorithm for two-body $b$-hadron decays. In order to better refine the selection a multivariate BDT classifier (Boosted Decision Tree [5]) is used. The input variables used in the BDT algorithm consist of kinematic and topological information on the tracks and the $B$ meson, such as the transverse momentum, the IP, the distance of closest approach, the separation of the SV with respect to the primary vertex (PV) and the proper decay time of the $b$-hadron candidate. Essential in the selection is the use of the particle identification information which allows the separation of the events in mutually exclusive samples, depending on the particles in the final state $(K \pi, K p, p \pi, \pi \pi$ and $K K)$. The PID selection efficiency is evaluated following a data-driven method, which uses samples of well identified pions, kaons and protons from $D^{*+} \rightarrow$ $D^{0}\left(\rightarrow K^{-} \pi^{+}\right) \pi^{+}, \Lambda \rightarrow p \pi^{-}$and $\Lambda_{c}^{+} \rightarrow p \pi^{+} K^{-}$decays [6]. The final optimisation for the PID and BDT selections is separate for the $B_{s}^{0} \rightarrow \pi^{+} \pi^{-}$and $B^{0} \rightarrow K^{+} K^{-}$, in order to maximise the analysis sensitivity. For each of the two samples BDT and PID selection criteria are optimised simultaneously. The final selected samples are then fitted to extract the signal yields. The strategy 

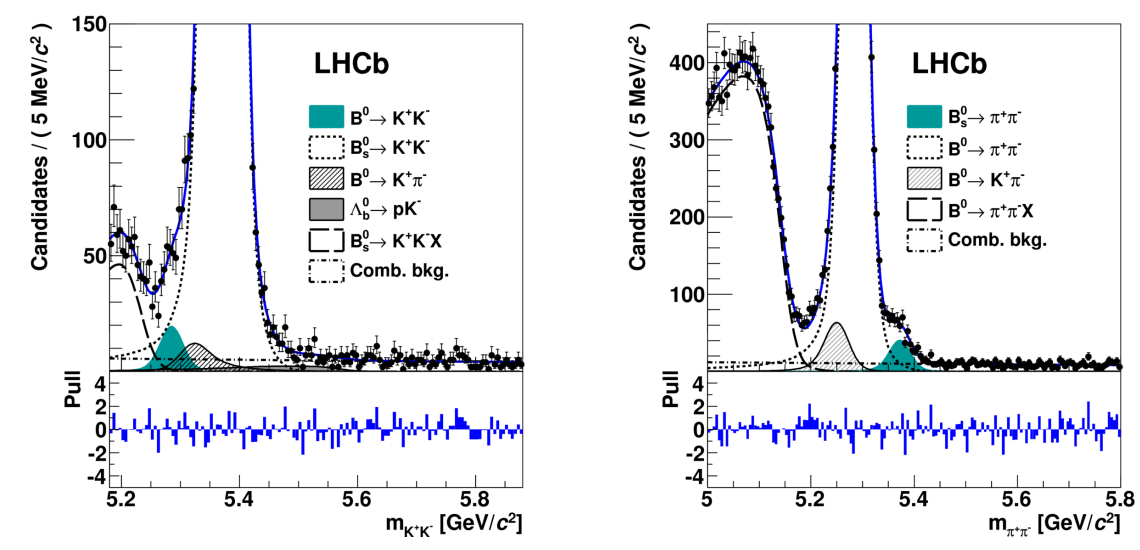

Figure 1: Distribution of the $K^{+} K^{-}$and the $\pi^{+} \pi^{-}$invariant masses and the fit projection superimposed. The two plain curves represent the signal decays.

used is a simultaneous two-body mass fit of the sub-samples. Figure 1 shows the distribution of the $K^{+} K^{-}$and the $\pi^{+} \pi^{-}$invariant masses and the fit projection superimposed. The two plain curves represent the fitted signal yields.

The main sources of systematic uncertainties are related to the choice of the fit model and to the determination of the PID efficiencies. The first uncertainty is evaluated as the difference in the signal yield when using the nominal or alternative fit models. The second systematic effect is accounted for by performing a set of pseudo-experiments where the PID efficiency is randomly varied; the standard deviation of the obtained signal yield distribution is used as uncertainty. The branching fractions are evaluated with respect to the $B^{0} \rightarrow K^{+} \pi^{-}$normalisation decay, using the HFAG average for its branching fraction [7]. The obtained values are:

$$
\begin{gathered}
\mathscr{B}\left(B^{0} \rightarrow K^{+} K^{-}\right)=(7.80 \pm 1.27(\text { stat }) \pm 0.81(\text { syst }) \pm 0.21(\text { norm })) \times 10^{-8} \\
\mathscr{B}\left(B_{s}^{0} \rightarrow \pi^{+} \pi^{-}\right)=\left(6.91 \pm 0.54(\text { stat }) \pm 0.63(\text { syst }) \pm 0.19(\text { norm }) \pm 0.40\left(f_{s} / f_{d}\right)\right) \times 10^{-7}
\end{gathered}
$$

where in the case of the $B_{s}^{0} \rightarrow \pi^{+} \pi^{-}$branching fraction the last uncertainty accounts for the ratio of $B_{s}^{0} / B^{0}$ production rates.

\section{Search for $B_{(s)}^{0} \rightarrow \phi \pi^{+} \pi^{-}$decays}

The $B_{(s)}^{0} \rightarrow \phi \pi^{+} \pi^{-}$decays have never been observed before. These decays proceed trough FCNC transitions, which provide a sensitive probe for the search of BSM physics since new particles may enter in the loops. The search is performed with a time-integrated and flavour-untagged analysis [8]. The sample selection is performed in two steps. At a first stage well-reconstructed tracks are selected, and are requested to have a large IP with respect to any PV in the event, and to be consistent with coming from a displaced SV. Two kaons having opposite charge and coming from a common vertex are combined to form the $\phi$ candidate. Pion pairs are selected in the mass range $400-1600 \mathrm{MeV} / \mathrm{c}^{2}$. The $\phi$ and the pion pairs are then combined to form $B_{s}^{0}$ or $B^{0}$ meson candidates. A further requirement on the flight distance of the $B$ candidates, ensuring their origin from the PV, helps to better separate the signal from the combinatorial background. The second 

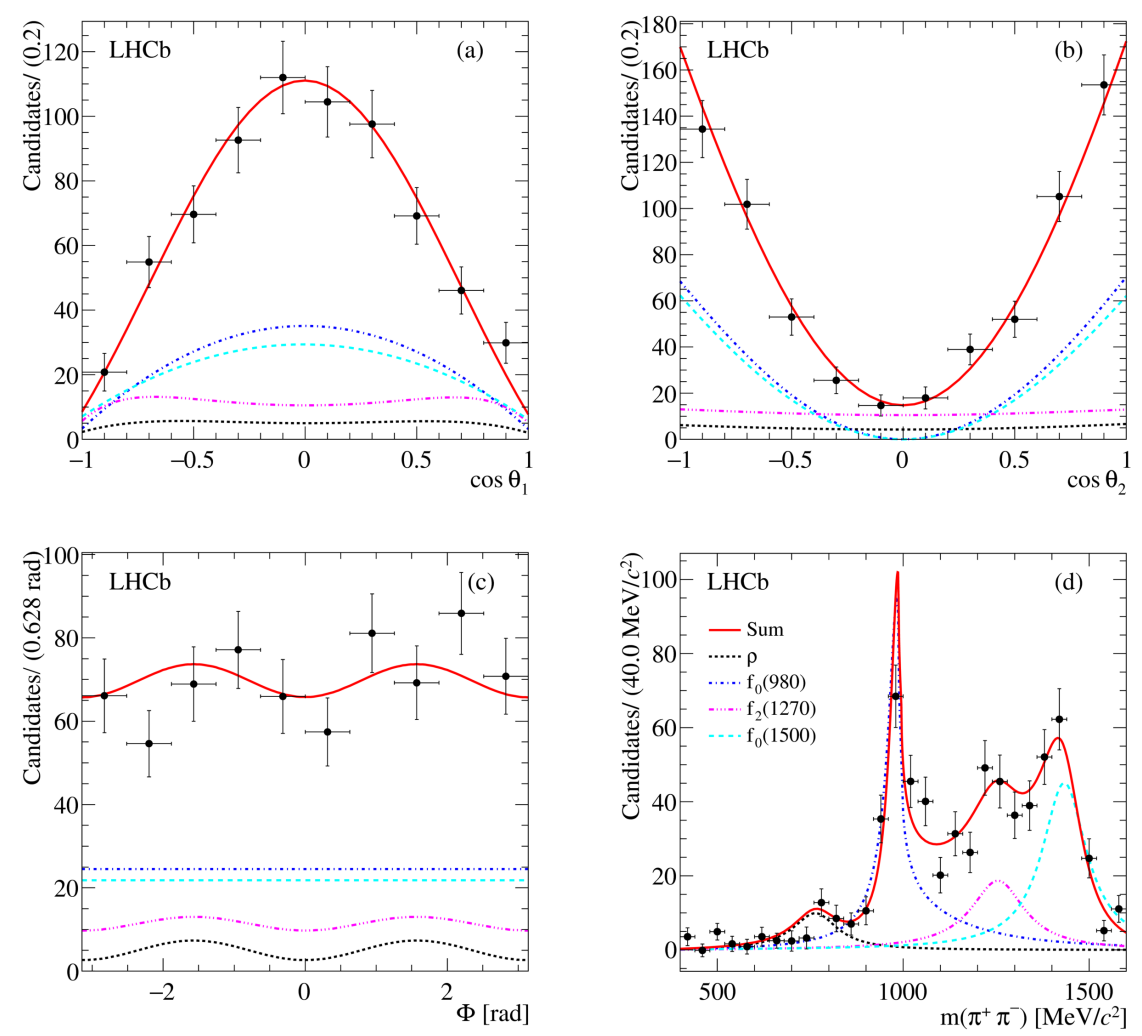

Figure 2: Projections of (a) $\cos \theta_{1}$, (b) $\cos \theta_{2}$, (c) $\Phi$, and (d) $m_{\pi^{+} \pi^{-}}$distributions. $\theta_{1}$ is the angle between the $\pi^{+}$direction in the $\pi^{+} \pi^{-}$rest frame and the $\pi^{+} \pi^{-}$direction in the $B$ rest frame, $\theta_{2}$ is the angle between the $K^{+}$direction in the $\phi$ rest frame and the $\phi$ direction in the $B$ rest frame, and $\Phi$ is the angle between the $\pi^{+} \pi^{-}$ system and the $\phi$ meson decay planes. The $\rho^{0}$ contribution is shown by the dotted (black) curve, the $f_{0}(980)$ by the dot-dashed (blue) curve, the $f_{2}(1270)$ by the double-dot-dashed (magenta) curve and the $f_{0}(1500)$ by the dashed (cyan) curve.

step of the selection uses a BDT algorithm based on kinematic variables of the $B$ meson and its decay products, kaon PID and information on the vertex displacement with respect to the PV. The signal yields are then extracted from a one-dimensional fit to the $\phi \pi^{+} \pi^{-}$invariant mass. A first observation is obtained for the $B_{s}^{0} \rightarrow \phi \pi^{+} \pi^{-}$mode, while only a first evidence is obtained for the $B^{0} \rightarrow \phi \pi^{+} \pi^{-}$decay with a significance of $4.5 \sigma$.

The $\mathscr{B}\left(B_{s}^{0} \rightarrow \phi \pi^{+} \pi^{-}\right)$in the invariant-mass range $400<m_{\pi^{+} \pi^{-}}<1600 \mathrm{MeV} / \mathrm{c}^{2}$ is measured to be

$$
\mathscr{B}\left(B_{s}^{0} \rightarrow \phi \pi^{+} \pi^{-}\right)=(1.82 \pm 0.25 \pm 0.41 \pm 0.14) \times 10^{-7},
$$

where the first uncertainty is statistical, the second is systematic and the third is due to the $B_{s}^{0} \rightarrow \phi \phi$ mode used as normalisation channel. Using the $s$ Plot technique [17], with the $b$-hadron candidate invariant mass used as the control variable, the $B_{s}^{0} \rightarrow \phi \pi^{+} \pi^{-}$signal is selected and an amplitude analysis is performed in order to identify the resonance contributions. A four-dimensional fit of $m_{\pi^{+} \pi^{-}}$mass and decay angle distributions of the $B_{s}^{0}$ candidates is performed. Figure 2 shows the fit results. The main contributions are given by the $B_{s}^{0} \rightarrow \phi f_{0}(980)$ decay, which is observed with a 
significance of $8 \sigma$, and the $B_{s}^{0} \rightarrow \phi f_{2}(1270)$ decay, with a significance of $5 \sigma$. An evidence $(4 \sigma)$ is also set for the $B_{s}^{0} \rightarrow \phi \rho^{0}$ mode. This last result, even if still compatible with the SM prediction, is lower than expected [8]. The resulting branching fractions are measured to be:

$$
\begin{aligned}
\mathscr{B}\left(B_{s}^{0} \rightarrow \phi f_{0}(980)\right) & =\left(1.12 \pm 0.16_{-0.08}^{+0.09} \pm 0.1\right) \times 10^{-6} \\
\mathscr{B}\left(B_{s}^{0} \rightarrow \phi f_{2}(1270)\right) & =\left(0.61 \pm 0.13_{-0.05}^{+0.12} \pm 0.06\right) \times 10^{-6} \\
\mathscr{B}\left(B_{s}^{0} \rightarrow \phi \rho^{0}\right) & =(2.7 \pm 0.7 \pm 0.2 \pm 0.2) \times 10^{-7}
\end{aligned}
$$

In the upper region of the $m_{\pi^{+} \pi^{-}}$invariant mass $\left(1350-1600 \mathrm{MeV} / \mathrm{c}^{2}\right)$ a peaking contribution is observed, and is identified to be due to the superposition of the $f_{0}(1500)$ and the $f_{0}(1370)$ resonances.

\section{Search for the $B_{s}^{0} \rightarrow \phi \eta^{\prime}$ decay}

The $B_{s}^{0} \rightarrow \phi \eta^{\prime}$ decay, which proceeds predominantly through $b \rightarrow s \bar{s} s$ transitions, has never been observed. The range of predictions for its branching fraction goes typically from $0.1 \times 10^{-6}$ to $20 \times 10^{-6}$ with large uncertainties [9][10][11][12][13][14]. This is due to a poor knowledge of the form factors, penguin contributions and the $\omega-\phi$ mixing angle. The search is performed using the $B^{+} \rightarrow \eta^{\prime} K^{+}$decay as normalisation channel in the determination of the branching fraction [15]. After a trigger selection at hardware and software stages, only tracks originated from a displaced vertex and identified as pions or kaons are kept. Two oppositely charged pions, with invariant mass larger then $520 \mathrm{MeV} / \mathrm{c}^{2}$ and with a common vertex are combined with a good quality photon to form an $\eta^{\prime}$ meson, while the $\phi$ is formed with two oppositely charged kaons in a mass window of $30 \mathrm{MeV} / \mathrm{c}^{2}$ centred on its nominal mass [16]. The $\eta^{\prime}$ and $\phi$ candidates are finally combined to form a $B_{s}$ meson. A BDT is used to further separate signal and background. The algorithm uses kinematical and topological variables, among which the most powerful are the vertex quality of the $B$ candidate and the transverse momentum of the photon. The signal yield is established trough a simultaneous two-dimensional fit of the $B_{s}$ and the $\eta^{\prime}$ invariant masses of the $B_{s}^{0} \rightarrow \phi \eta^{\prime}$ and $B^{+} \rightarrow \eta^{\prime} K^{+}$samples. The fit model for $B_{s}^{0} \rightarrow \phi \eta^{\prime}$ includes a shape to account for the physics background $B_{s}^{0} \rightarrow \phi \phi$, where one of the two $\phi$ mesons decays in the $\pi^{+} \pi^{-} \pi^{0}$ final state. Figure 3 shows the mass distributions observed in data with fit projections overlaid. No signal is found. The fitted signal yield is $-3.2_{-3.8}^{+5.0}$. Upper limits are set for the branching fraction using a Bayesian approach and assuming a uniform prior. The measured upper limit (including the contribution of the systematic uncertainty) is:

$$
\mathscr{B}\left(B_{s}^{0} \rightarrow \phi \eta^{\prime}\right)<0.82(1.01) \times 10^{-6} \text { at } 90 \%(95 \%) \mathrm{CL} .
$$

\section{Conclusion}

Three recent results for charmless two-body and quasi-two-body $B$ meson decays are presented. These measurements lead to the first observation of the decays $B^{0} \rightarrow K^{+} K^{-}, B_{s}^{0} \rightarrow \phi f_{0}(980)$ and $B_{s}^{0} \rightarrow \phi f_{2}(1270)$. An upper limit is set for the $B_{s}^{0} \rightarrow \phi \eta^{\prime}$ decay, which is incompatible with 

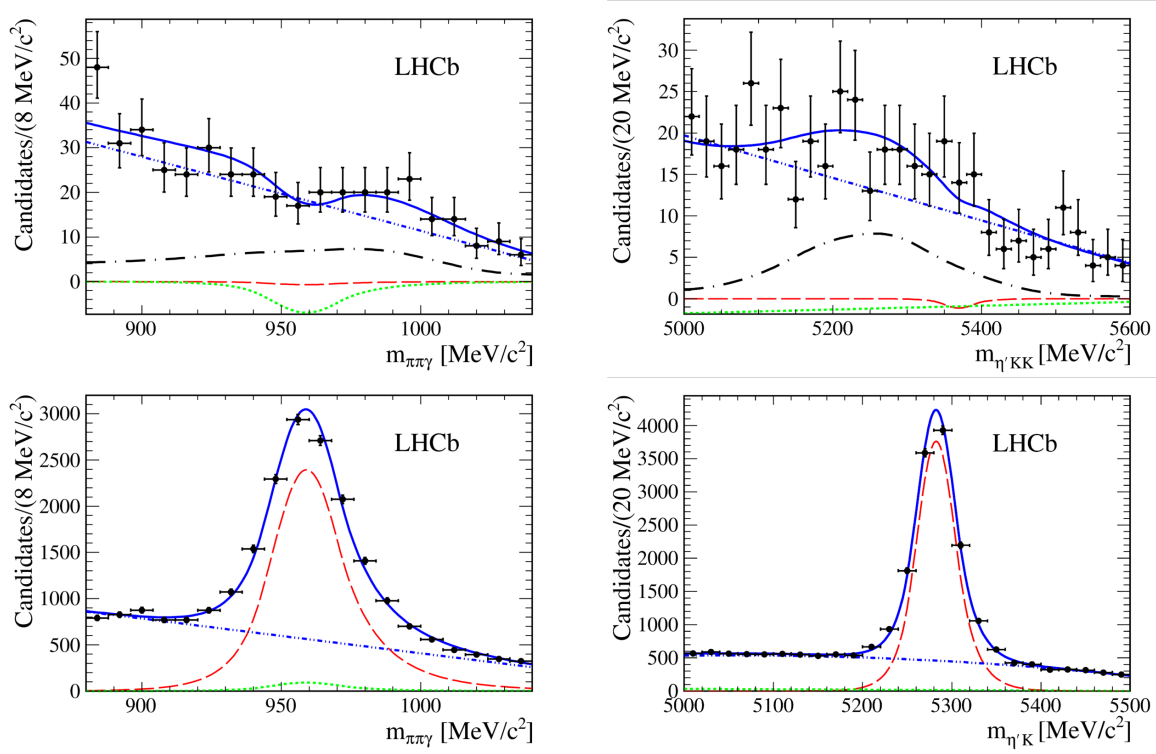

Figure 3: Distributions of the (left) $\pi^{+} \pi^{-} \gamma$ and (right) $\eta^{\prime} K^{+} K^{-}$massed for the (top) $B_{s}^{0} \rightarrow \phi \eta^{\prime}$ and (bottom) $B^{+} \rightarrow \eta^{\prime} K^{+}$candidates with the fit projections overlaid. The signal yields are indicated with red dashed curves.

the central values of many predictions, although the large uncertainties on these predictions do not exclude them definitively.

\section{References}

[1] H-Y Cheng and J.G. Smith, Charmless Hadronic B Meson Decays, Annual Review of Nuclear and Particle Science 59 215-243 (2009).

[2] Aaij, R. et al. [LHCb collaboration], Measurement of $\sigma(p p \rightarrow b \bar{b} X)$ at $\sqrt{s}=7 \mathrm{TeV}$ in the forward region, Phys. Lett. B694 209 (2010) [hep-ex/1009.2731].

[3] Aaij, R. et al. [LHCb collaboration], Observation of the annihilation decay mode $B^{0} \rightarrow K^{+} K^{-}$, [hep-ex/1610.08288].

[4] Aaij, R. et al. [LHCb collaboration], Measurement of b-hadron branching fractions for two-body decays into charmless charged hadrons, JHEP 10037 (2012) [hep-ex/1206.2794].

[5] L. Breiman, J. H. Friedman, R. A. Olshen, and C. J. Stone, Classification and regression trees, Wadsworth international group, Belmont, California, USA, 1984.

[6] M. Adinolfi et al., Performance of the LHCb RICH detector at the LHC, Eur. Phys. J. C73 2431 (2013) [hep-ex/1211.6759].

[7] Y. Amhis et al., [Heavy Flavor Averaging Group], Averages of b-hadron, c-hadron, and $\tau$-lepton properties as of summer 2016, [hep-ex/1612.07233].

[8] Roel Aaij et al. [LHCb collaboration], Observation of the decay $B_{s}^{0} \rightarrow \phi \pi^{+} \pi^{-}$and evidence for $B^{0} \rightarrow \phi \pi^{+} \pi^{-}$, Phys. Rev. D95 012006 (2017) [hep-ex/1610.05187] .

[9] H.-Y. Cheng and C.-K. Chua, QCD factorization for charmless hadronic $B_{s}^{0}$ decays revisited, Phys. Rev. D80 114026 (2009), [hep-ph / 0910 . 5237]. 
[10] A. Ali et al, Charmless non-leptonic $B_{s}$ decays to PP, PV and VV final states in the pQCD approach, Phys. Rev. D76 074018 (2007), [hep-ph/ 0703162$].$

[11] X.-f. Chen, D.-q. Guo, and Z.-j. Xiao, $B_{s}^{0} \rightarrow(\rho, \omega, \phi) \eta^{(\prime)}$ decays in the perturbative QCD approach, [hep-ph/0701146].

[12] W. Wang, Y.-M. Wang, D.-S. Yang, and C.-D. Lü, Charmless two-body $B_{s}^{0} \rightarrow P V$ decays in soft collinear effective theory, Phys. Rev. D78 034011 (2008), [hep-ph/ 0801 . 3123].

[13] H.-Y. Cheng, C.-W. Chiang, and A.-L. Kuo, Updating $B \rightarrow P P, V P$ decays in the framework of flavor symmetry, Phys. Rev. D91 014011 (2015), [hep-ph/1409.5026].

[14] S.-H. Zhou, Q.-A. Zhang, W.-R. Lyu, and C.-D. Lü, Analysis of charmless two-body B decays in factorization assisted topological amplitude approach, [hep-ph/11608. 02819].

[15] Roel Aaij et al. [LHCb collaboration], Search for the $B_{s}^{0} \rightarrow \phi \eta^{\prime}$ decay, [hep-ex/1612 . 08110].

[16] C. Patrignani et al. [Particle Data Group], Review of particle physics, Chin. Phys. C40 100001 (2016).

[17] M. Pivk and F. R. Le Diberder, sPlot: A statistical tool to unfold data distributions, Nucl. Instrum. Methods Phys. Res., Sect. A555, 356 (2005). 